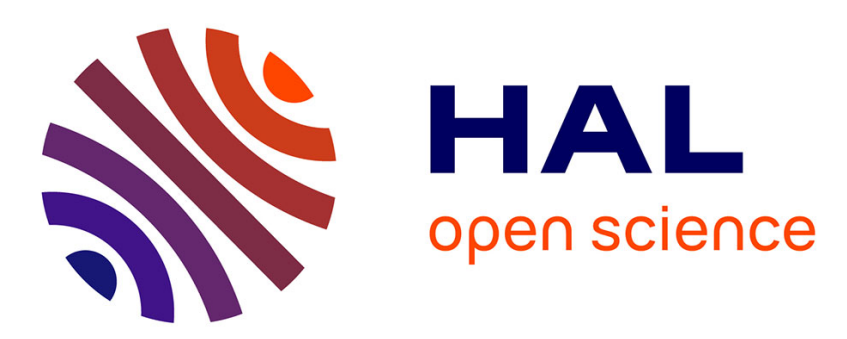

\title{
COMMUNICATION ET ACCUMULATION DU CAPITAL Pour une perspective de longue durée Serge Walery
}

\section{To cite this version:}

Serge Walery. COMMUNICATION ET ACCUMULATION DU CAPITAL Pour une perspective de longue durée. Quaderni, 1990, Finance et technologie de la communication, 12 (1), pp.15-35. 10.3406/quad.1990.1914 . hal-01212109

\section{HAL Id: hal-01212109 \\ https://hal-amu.archives-ouvertes.fr/hal-01212109}

Submitted on 6 Oct 2015

HAL is a multi-disciplinary open access archive for the deposit and dissemination of scientific research documents, whether they are published or not. The documents may come from teaching and research institutions in France or abroad, or from public or private research centers.
L'archive ouverte pluridisciplinaire HAL, est destinée au dépôt et à la diffusion de documents scientifiques de niveau recherche, publiés ou non, émanant des établissements d'enseignement et de recherche français ou étrangers, des laboratoires publics ou privés. 


\section{COMMUNICATION ET ACCUMULATION DU CAPITAL Pour une perspective de longue durée}

Références: Serge WALERY. Communication et accumulation du capital : pour une perspective de longue durée. QUADERNI, n¹2, Hiver 1990/1991, pp 15-35.

Pour bon nombre d'observateurs contemporains, le développement, tant qualitatif que quantitatif, que connaît depuis quelques années le procès ${ }^{1}$ de communication, serait porteur d'une transformation de la circulation économique, ${ }^{2}$ voire du mode d'accumulation du capital.

Si la simple observation de la réalité conduit effectivement à constater que les pratiques concrètes de la circulation économique sont modifiées par le développement actuel du procès de communication, l'appréciation de la portée de ces transformations quant à la nature de la circulation économique et à sa "place" ou son "rôle" dans le mode d'accumulation du capital est, elle, l'objet d'un débat, malheureusement bien trop souvent implicite. De façon très schématique, on peut distinguer trois grandes catégories d'interprétations de la portée des transformations de la circulation économique induites par le développement du procès de communication :

- Ces transformations ne modifient pas de façon significative la nature de la circulation économique, et ne constituent donc qu'un phénomène anecdotique du point de vue du mode d'accumulation du capital et du développement capitaliste. On remarque bien sûr, que, personne ne songeant à se fixer un objet de recherche anecdotique, peu de discours contemporains sur la communication se réfèrent à une telle interprétation.

- Ces transformations constituent la manifestation concrète d'une redéfinition de la nature et du rôle de la circulation économique ; elles apparaissent ainsi comme l'indice d'une remise en cause des modalités existantes de l'accumulation du capital.

- Ces transformations sont l'indice d'une remise en cause, non seulement des modalités, mais de la nature même de l'accumulation du capital, qui tendrait désormais à être concurrencée par l'essor d'une nouvelle dynamique, celle de la "société de communication"...

La dernière interprétation, aussi lourde de conséquences soit-elle, se trouve en filigrane derrière un nombre croissant de discours sur le procès de communication ; elle est même explicitement revendiquée par certains. C'est le cas des nombreux prophètes de la "société de communication", au premier rang desquels on trouve, bien sûr, ceux qui pensent participer activement à l'émergence de cette "nouvelle société". Ainsi, selon François Henri de Virieu $^{3}$ :

«Presque toutes les grandes institutions de la société, presque toutes les activités humaines changent aujourd'hui de logique de fonctionnement pour se plier à la logique du système médiatique. ( ... ) Les médias ne sont plus de simples collecteurs, trieurs, transporteurs et diffuseurs d'informations. Ils constituent par leur existence même, par l'étendue de leurs performances, un

\footnotetext{
${ }^{1}$ On se réfère ici à la définition de Michel Aglietta. Procès : ensemble organisé d'activités soumises à une logique générale. Voir : Régulation et crises du capitalisme. L'expérience des Etats-Unis. Paris. Calmann-Lévy. 1976. Page 133.

2 i.e : la circulation au sein de l'organisation économique : flux de biens et services, flux de revenus, flux financiers, et bien sûr, flux d'informations.

${ }^{3}$ F.H. de Virieu. La médiacratie. Paris, Flammarion, 1990. pp 15, 19.
} 
nouveau principe organisateur de notre vie sociale.»

De façon plus surprenante, certains économistes ou spécialistes des organisations n'hésitent pas à pronostiquer « la fin d'une tradition de société industrielle d'accumulation capitaliste et de gestion libérale.» ${ }^{4}$ Pour eux, le développement des techniques d'information et de communication constitue bien la manifestation de l'émergence d'un nouvel ordre social. Ainsi, selon Guy Terny ${ }^{5}$ :

"Information et communication ne sauraient être traitées comme une nouvelle industrie, parmi d'autres, relevant des mêmes modèles d'analyse. En participant à une mutation des conditions de la production des biens et des services, du commerce et des relations entre les hommes, elles concourent à la naissance d'un nouveau type de société dont les contours se dessinent mais restent encore imparfaitement prévisibles.»

Dans une perspective historique de longue durée ${ }^{6}$, de telles affirmations ont une portée considérable. Accepter l'idée, qu'avec le développement contemporain du procès de communication, une nouvelle logique d'organisation sociale tend à se mettre en place, c'est considérer, au moins implicitement, que s'annonce le déclin de la logique jusqu'ici dominante, celle du procès d'accumulation du capital.

Il serait absurde de prétendre, en quelques pages, confirmer ou infirmer l'idée de l'émergence d'une "société de communication" ; la réflexion en termes de longue durée, impose une grande modestie des objectifs. On se bornera donc, en s'appuyant sur certains résultats de travaux antérieurs en la matière, ${ }^{7}$ à fournir quelques éléments susceptibles d'éclairer un peu cette problématique, du point de vue de la longue durée ; on s'efforcera, en présentant certains résultats de l'analyse de longue durée des mouvements du procès de communication, du procès d'accumulation du capital et de leur articulation (ou interférences), d'indiquer ce que pourrait être une analyse, en termes de longue durée, du développement contemporain du procès de communication.

Sans pour autant nier l'intérêt d'une telle interprétation, on tentera de montrer que ceux qui proclament l'avènement de la "société de communication" font sans doute preuve d'un peu de précipitation ; dans la mesure où l'idée selon laquelle le développement actuel du procès de communication serait la traduction de l'émergence d'une "société de communication" suppose une transformation radicale (dans une perspective de longue durée) de l'articulation procès de communication-procès d'accumulation du capital, les arguments jusqu'ici présentés par les prophètes de la "société de communication" apparaissent largement insuffisants, puisqu'ils ignorent presque systématiquement la problématique de longue durée. Du point de vue de la longue durée, l'émergence d'une "société de communication" n'est pas, a priori, une éventualité absurde, mais l'état actuel des recherches sur le procès de communication interdit de considérer cette possibilité autrement que comme une pure hypothèse d'école.

Comme il ne saurait être question d'aborder ici l'ensemble des mouvements de longue durée de l'articulation procès de communication-procès d'accumulation du capital, il convient

\footnotetext{
${ }^{4}$ R. Sainsaulieu. In : Actes du colloque de l'ENSPTT des 28-29 novembre 1988. Paris, ENSPTT, 1989, p 53.

${ }^{5}$ G. Terny. In : Actes du colloque de l'ENSPTT des 28-29 nov 1988. Op cit. p 14.

${ }^{6}$ Sur la notion de longue durée, voir (bien sûr !) Fernand Braudel :

- La longue durée. Annales E.S.C. № 4, Oct-déc 1958, p 725-753.

- Civilisation matérielle, économie et capitalisme (XV-XVIII siècles). 3 volumes, Paris, Armand Colin, 1979, Tome 3, pp 56-70

7 S. Walery. Contrôle du temps et organisation des activités économiques : analyse de mouvements économiques de longue durée. Thèse d'État en Sciences Économiques, Université Paris-9-Dauphine, 1987.
} 
de réduire très sensiblement le champ d'analyse. Ne disposant que de quelques pages, on se limitera à l'étude d'un détail du "tableau".

En premier lieu, il convient de renoncer à traiter l'ensemble de la période historique caractérisée par la prééminence du procès d'accumulation du capital dans le mouvement social, c'est à dire, par la dynamique du capitalisme. ${ }^{8}$ Afin de mieux souligner les spécificités de la problématique de longue durée, on s'intéressera à la sous-période la plus ancienne, celle qui correspond aux origines du capitalisme, à cette époque où, loin de la puissance qu'elle allait acquérir par la suite, la dynamique du capitalisme n'était encore qu'un mouvement social localisé, mais déjà moteur de l'évolution économique et sociale. Rappelons que cette époque, qui couvre approximativement les $14^{\text {ème }}, 15^{\text {ème }}$ et $16^{\text {ème }}$ siècles, correspond au premier "âge" du capitalisme commercial, celui des hommes d'affaires ; aux $17^{\text {ème }}$ et $18^{\text {ème }}$ siècles, le second "âge" sera celui des compagnies et des Etats ; bien sûr, la fin du $18^{\text {ème }}$ et le début du $19^{\text {ème }}$ siècles seront marqués par la transition du capitalisme commercial au capitalisme industriel, et par une montée en puissance considérable de la dynamique du capitalisme. $^{9}$

Même sur une période limitée, l'articulation procès de communication-procès d'accumulation du capital ne saurait être appréhendée ici, dans son ensemble ; il convient donc de se limiter à l'étude d'un élément spécifique de cette articulation. Du fait que, pour de nombreux observateurs contemporains, l'extraordinaire accélération des communications à laquelle nous assistons depuis quelques années constitue un bouleversement majeur, on s'intéressera, en priorité, à la vitesse des communications, sans pour autant pouvoir l'isoler de la tendance au développement des réseaux.

Finalement, on se limitera donc à montrer comment, dès les origines du capitalisme, l'accroissement de la vitesse des communications et le développement des réseaux ont constitué deux mouvements induits par les nécessités de l'accumulation du capital, et qui, en retour, ont nourri le développement de l'accumulation. En d'autres termes, ces deux mouvements furent étroitement déterminés par la dynamique du capitalisme telle qu'elle se matérialisait à l'époque considérée. On en déduira, moyennant quelques précautions, que l'accélération des communications et le développement des réseaux ne peuvent être présentés comme des mouvements autonomes, et, a fortiori, comme des mouvements porteurs d'une dynamique concurrente de celle du capitalisme, que si cette présentation s'appuie sur une argumentation extrêmement solide, en matière de mouvements de longue durée.

\section{Capitalisme commercial et communications : une problématique}

La première période du capitalisme, le capitalisme commercial, débuta aux $14^{\text {ème }}$, $15^{\text {ème }}$ et $16^{\text {ème }}$ siècle, avec la constitution progressive d'un groupe de grands marchands ou d'hommes d'affaires, se distinguant de plus en plus nettement du commun des marchands de l'époque. N'ayant pas de boutique, rompant avec la traditionnelle spécialisation des activités commerciales, les hommes d'affaires de la Renaissance n'avaient comme seule préoccupation, que la réalisation de gains commerciaux. Comme le soulignait Fernand Braudel : ${ }^{10}$

«La recherche du profit, la maximisation du profit sont déjà les règles implicites du capitalisme en ce temps-là.»

\footnotetext{
${ }^{8}$ F. Braudel. La dynamique du capitalisme. Paris. Arthaud. 1985.

${ }^{9}$ Sur cette périodisation, voir (entre autres) :

- F. Braudel, 1979. Op cit.

- M. Beaud. Histoire du capitalisme de 1500 à nos jours. Paris. Seuil. 1981.

${ }^{10}$ F. Braudel, 1979. Op cit, vol 2, p 216.
} 
C'est pourquoi les hommes d'affaires de la Renaissance privilégièrent le commerce au loin, qui, malgré les risques encourus, était source de gains considérables, et s'efforcèrent, jouant au besoin de leur situation de créditeurs des princes, de bénéficier, chaque fois que possible, de situations monopolistiques. Leurs compagnies, le plus souvent à caractère familial, avaient des succursales dans toutes les grandes places commerciales européennes, afin de mieux profiter des éventuelles opportunités; leur horizon ne connaissait pas de frontières.

Dès les premiers balbutiements du capitalisme commercial, le degré de développement des systèmes de communication devint une préoccupation permanente dans l'application concrète de la logique d'accumulation du capital. Entre l'espace du capitalisme commercial (i.e : l'horizon spatial des hommes d'affaires de la Renaissance), qui s'apparente à celui de l'ère moderne, et les moyens de communication de l'époque, si peu différents de ceux du Moyen Âge, existait, du point de vue de l'accumulation du capital, une inadéquation totale.

\section{$\underline{\text { Lenteur et irrégularité des communications }}$}

Au moment des grandes découvertes, les moyens de communication n'avaient que peu évolué depuis la révolution technique du début du second millénaire. Bien sûr, les routes et l'organisation des transports terrestres s'étaient quelque peu améliorées ; les navires et les techniques de navigation avaient sensiblement progressé ; il n'en reste pas moins que la vitesse et la régularité des transports n'avaient que peu évolué.

"Jusqu'au $18^{\text {ème }}$ siècle, les navigations sont interminables, les transports terrestres comme paralysés.»11

Malgré les efforts des hommes d'affaires pour améliorer l'efficacité des services postaux, au début du $16^{\text {ème }}$ siècle, un courrier appartenant à un réseau organisé couvrait en moyenne 10 lieues par jour s'il était à pied, et 15 à 20 lieues s'il était à cheval. ${ }^{12} \mathrm{Il}$ ne s'agit cependant que d'une moyenne ; en fonction des conditions et des imprévus du voyage, les temps effectifs étaient extrêmement variables. Ainsi, pour qui avait les moyens de recourir à un service postal efficace, le temps de transmission d'une nouvelle au départ de Venise s'établissait entre 2 et 15 jours pour Gênes, entre 8 et 36 jours pour Anvers, et entre 27 et 69 jours pour Lisbonne (les temps moyens respectifs étant de une semaine, trois semaines, et un mois et demi). ${ }^{13}$ On imagine donc ce que pouvait être la durée des voyages pour les courriers ne disposant pas de relais et d'abris à intervalles réguliers, et, à plus forte raison, pour les transports terrestres de marchandises.

Lenteur et irrégularité caractérisaient, de la même façon, le transport maritime. A la fin du $15^{\text {ème }}$ siècle, le voyage Gênes-Sicile et retour nécessitait entre 30 et 70 jours, l'aller-retour de Gênes en Flandre par Gibraltar et l'Atlantique généralement plus d'un an. ${ }^{14}$ Au début du $16^{\text {ème }}$ siècle, le trajet de Venise à Constantinople demandait entre 15 et 81 jours, celui de Venise à Alexandrie entre 17 et 89 jours. $^{15}$

\footnotetext{
${ }^{11}$ F. Braudel, 1979. Op cit, vol 1, p 365.

${ }^{12}$ V. Vasquez de Prada. Lettres marchandes d'Anvers. 4 volumes, Paris, S.E.V.P.E.N, 1960-1961. Tome 1.

${ }^{13}$ P. Sardella. Nouvelles et spéculations à Venise au début du $16^{\text {ème }}$ siècle. Paris, Armand Colin, 1948, pp 56-57.

14 J. Heers. Gênes au $X V^{\circ}$ siècle : civilisation méditerranéenne, grand capitalisme et capitalisme populaire. Paris, Flammarion, 1971, pp 296-297.

${ }^{15}$ P. Sardella, 1948. Op cit.
} 
De nouveaux horizons : l'espace du capitalisme commercial

Cependant, alors que les moyens de communication ne progressaient que de façon marginale, les perspectives de profit offertes par le commerce à long rayon d'action élargirent considérablement l'horizon spatial des hommes d'affaires de la Renaissance, ainsi que l'espace de leurs activités. En effet, même si les quantités concernées ne furent longtemps qu'assez faibles, les profits du commerce au loin étaient tels, que celui-ci devint le champ privilégié de l'accumulation du capital.

"Le commerce au loin a sans doute tenu le premier rôle dans la genèse du capitalisme marchand, et il en fut longtemps l'ossature.» ${ }^{16}$

«Le marché à l'échelle mondiale devient le vecteur dominant de l'évolution économique.» ${ }^{17}$

C'est dans ce cadre que les hommes d'affaires investirent rapidement les nouveaux horizons mis à jour du $13^{\text {ème }}$ au $16^{\text {ème }}$ siècle, étendant ainsi, et de façon considérable, l'espace de la circulation économique à caractère capitaliste.

Dès la fin du $13^{\text {eme }}$ siècle, l'espace commercial occidental fut bouleversé par la création de nouvelles routes terrestres et maritimes reliant les deux grands centres économiques de l'époque, l'Italie du Nord et les Pays-Bas. D'une part, l'ouverture des routes alpines du Simplon, du Saint-Gothard et du Brenner provoqua un important basculement vers l'est, dont l'une des conséquences les plus connues fut de favoriser l'essor du sud de l'Allemagne au détriment des foires de Champagne. D'autre part, le franchissement du détroit de Gibraltar provoqua la réunion de deux univers maritimes jusque là distincts, l'atlantique et le méditerranéen, ouvrant ainsi aux navires génois, et plus tard vénitiens, la route de Londres et de Bruges, pour le plus grand profit de la Péninsule Ibérique, frontière entre ces deux univers désormais réunis. Par ailleurs, la fin du Moyen Âge vit également les marchands italiens, et en particulier vénitiens, réactiver les liaisons maritimes avec l'Orient méditerranéen, (Constantinople, Alexandrie, Beyrouth...) intermédiaire incontournable pour le commerce si lucratif des produits d'Asie.

Ce n'est cependant qu'au $15^{\text {ème }}$ siècle, avec la lente progression des marins portugais d'Henri le Navigateur le long de la côte africaine, l'ouverture de la route des Indes et de l'Extrême-Orient par Barthélémy Dias, et, bien sûr, la découverte de ce qui allait devenir l'Amérique, que l'espace du capitalisme commercial connut sa mutation la plus brutale. Désormais, l'horizon des hommes d'affaires serait la planète entière.

\section{$\underline{\text { Capitalisme commercial et vitesse des communications }}$}

Si le développement du capitalisme commercial s'accompagna d'un accroissement considérable de l'espace d'activités des hommes d'affaires, il se traduisit également par une transformation radicale de l'appréhension du temps au sein du groupe social considéré, c'est à dire, par l'émergence d'une nouvelle logique de contrôle du temps. Pour les hommes d'affaires de la Renaissance, le rapport au temps était soumis aux nécessités de la maximisation des profits. Le temps était donc appréhendé en tant que déterminant des profits, selon les principes de l'équivalence temps-argent ; les hommes d'affaires inventèrent ainsi une véritable "économie marchande du temps". ${ }^{18}$

\footnotetext{
${ }^{16}$ F. Braudel, 1979. Op cit. vol 2, p 355.

${ }^{17}$ V.M. Godinho. Les découvertes $15^{\text {ème }}-16^{\text {ème }}$ : une révolution des mentalités. Paris, Éditions Autrement. Série "Mémoires", Supplément au n 1, 1990, pp 8-9.

${ }^{18}$ S. Walery, 1987. Op cit.
} 
Dans la mesure où, pour les hommes d'affaires, tout devait être soumis aux nécessités de la maximisation des profits, et donc de la rentabilisation du temps, la relation fondamentale entre l'espace et le temps fut, pour eux, la vitesse. Or, le procès de communication, s'inscrit dans l'espace et dans le temps. La vitesse des communications fut donc, dès les origines, un enjeu essentiel dans la perspective du développement capitaliste.

\section{Vitesse des communications et vitesse de rotation du capital}

\section{$\underline{\text { Capitalisme commercial et vitesse de rotation du capital }}$}

Du fait des perspectives de profit offertes par le commerce au loin et du degré de développement des moyens de communication, les circuits commerciaux organisés par les hommes d'affaires étaient extrêmement longs. Par exemple, ${ }^{19}$ au début du $16^{\text {ème }}$ siècle, le marchand vénitien Michiel da Lezze mit sur pied un trafic entre le Levant et l'Angleterre ; la galère chargeait des clous de girofle à Alexandrie, les transportait à Londres où ils étaient revendus, et où on chargeait des draps de laine à destination du Levant. La durée prévisible d'un tel voyage était de l'ordre d'un an ; mais, rappelons-le une fois de plus, l'imprévisible était la règle... Encore s'agissait-il d'un circuit binaire très simple ; or, les hommes d'affaires de la Renaissance recoururent fréquemment aux trafics triangulaires voire quadrangulaires. Ainsi, en 1505, le même Michiel da Lezze commandita une galère, devant quitter Venise chargée de mocenighi d'argent. À Tunis, l'argent devait être changé contre de la poudre d'or. A Valence la poudre d'or serait fondue et transformée en pièces à l'Hôtel des Monnaies de la ville. Dès lors, selon les aléas de la conjoncture, on ramènerait à Venise, soit les pièces elles-mêmes, soit une cargaison de laine... Plus généralement, avec le changement d'échelle provoqué par les grandes découvertes, la durée des circuits commerciaux allait atteindre un maximum ; par la suite, elle ne cesserait de diminuer.

" Jamais le monde n'a été aussi grand qu'au lendemain du périple de Magellan. ( ... ) L'univers né de la longue mutation des $X V^{\circ}$ et $X V I^{\circ}$ siècles a cinq ans. Cinq ans, c'est, en moyenne, le temps qu'il faut pour un aller-retour Espagne-Philippines. ${ }^{20}$

Pour le ou les hommes d'affaires qui avançaient les capitaux nécessaires à une opération commerciale au long cours, le moment crucial était le bouclage du circuit. C'est à cet instant ultime, et à cet instant seulement, que l'opération, c'est à dire le profit ou la perte, se réalisait. Or, entre l'amorce et le bouclage des circuits, bien des évènements pouvaient survenir. Bon nombre de circuits ne se bouclaient jamais ; accidents, naufrages, pirates, dégradations ou pertes de marchandises étaient alors monnaie courante. Et si on pouvait espérer éviter ces dangers majeurs, il n'était pas raisonnable de penser échapper à tous les autres risques du commerce au loin. Les voyages dont le déroulement était conforme aux prévisions constituaient l'exception ; délais, attentes et retards étaient la règle.

Cependant, chaque délai imprévu accroissait un peu plus la durée totale du circuit, c'est à dire, la durée d'immobilisation des capitaux engagés. Du point de vue des hommes d'affaires, chaque délai supplémentaire entraînait ainsi une réduction de la vitesse de rotation du capital, et constituait un frein supplémentaire à l'accumulation. La régularisation et la réduction de la durée des circuits commerciaux apparaissent ainsi, à l'époque du capitalisme commercial, comme une réponse aux nécessités de l'accumulation du capital.

\footnotetext{
${ }^{19}$ F. Braudel, 1979. Op cit. vol 2, p 118.

${ }^{20}$ P. Chaunu. Conquête et exploitation de nouveaux mondes (16 ${ }^{\text {ème }}$ siècle). Paris, P.U.F, Nouvelle Clio, 1969, p 277.
} 
$\underline{\text { La vitesse des communications comme déterminant de la vitesse de rotation du capital }}$

À l'époque considérée, la vitesse de rotation du capital (ou la durée des circuits) dépendait directement et indirectement de la vitesse des communications. D'une part, comme on l'a vu, le degré de développement des systèmes de communications rendait les phases de voyage très longues. D'autre part, pour de multiples raisons, les phases de transactions donnaient fréquemment lieu à des délais imprévus. Ainsi, la galère de Michiel da Lezze arrivant à Alexandrie, pouvait se trouvée confrontée à une suspension des exportations ou à une pénurie temporaire de clous de girofle, les quantités disponibles ayant été embarquées par d'autres marchands ; les prix pouvaient avoir augmenté dans des proportions telles, que la rentabilité de l'opération étant en cause, le capitaine de la galère se trouvait obliger d'en informer Venise, et d'attendre de nouvelles instructions... Or, tous ces problèmes résultaient, en dernier ressort, de la lenteur de la circulation des informations économiques. Ayant été prévenu à temps d'une importante variation du prix des clous de Girofle à Alexandrie, Michiel da Lezze aurait pu destiner sa galère à un autre port ou à un autre trafic, économisant ainsi plusieurs mois d'immobilisation des capitaux engagés. Plus généralement, qu'il s'agisse de marchandises, de monnaie, d'effets financiers ou d'informations, c'est toute la circulation économique capitaliste, qui souffrait en permanence de l'inefficacité des systèmes de communication.

"La lenteur des circuits est un mal général. Marchandises, argent comptant, lettres de change, voyagent dans un sens ou dans l'autre, se croisent, se rencontrent, s'attendent.» ${ }^{21}$

\section{$\underline{\text { Accumulation du capital et vitesse des communications }}$}

On constate finalement, que dans les conditions historiques considérées, la lenteur (et l'irrégularité) des communications, constituait un souci majeur pour les hommes d'affaires, un frein permanent à l'accumulation du capital, et une limite au développement du capitalisme. Ainsi s'établit, à l'ère du capitalisme commercial, une première relation entre le développement de l'accumulation du capital et l'évolution de la vitesse des communications : la logique d'accumulation du capital, qui, par nature, tend à l'accroissement de la vitesse de rotation du capital, poussait inéluctablement (du fait des conditions historiques concrètes de l'accumulation du capital) à l'accélération des communications.

D'ailleurs, même si, jusqu'au $18^{\text {ème }}$ siècle, l'évolution de la vitesse des communications restera modeste, dès les $15^{\text {ème }}$ et $16^{\text {ème }}$ siècles, les systèmes de communication les plus rapides et les plus efficaces sont ceux mis en place et organisés par les grandes maisons commerciales.

Au début du $16^{\text {ème }}$ siècle, les services de courrier des hommes d'affaires sont plus rapides que ceux des rois... Ainsi, l'empereur du Saint-Empire, Maximilien 1 d'Autriche, recourt fréquemment au réseau personnel de Jacob Fugger pour acheminer son propre courrier, et ce, malgré l'existence d'un service spécialement créé par les Comtes de Taxi, pour les besoins du gouvernement impérial. ${ }^{22}$ De même, au milieu du siècle, les courriers royaux espagnols devaient, en théorie, aller de Bruxelles à Madrid en une quinzaine de jours ; en réalité, il était exceptionnel que ces délais soient respectés. De leur côté, les courriers privés au service des hommes d'affaires faisaient généralement le même trajet en onze jours. En

\footnotetext{
${ }^{21}$ F. Braudel, 1979. Op cit. vol1, p 345.

${ }^{22}$ L. Schick. Un grand homme d'affaires du début du $16^{\text {ème }}$ siècle : Jacob Fugger. Paris, S.E.V.P.E.N, 1957.
} 
outre, alors qu'un courrier royal quittait Bruxelles toutes les trois semaines, les maisons commerciales organisaient, au minimum, un départ hebdomadaire. ${ }^{23}$

Même si les effets des efforts réalisés par les hommes d'affaires, en matière de vitesse de la circulation économique à caractère capitaliste, ne furent que marginaux jusqu'au $18^{\text {ème }}$ siècle, et même si le rythme d'évolution de la vitesse des communications dépend, par nature, de celui de l'histoire des techniques, il n'en reste pas moins qu'à la période considérée, l'accélération des communications ne peut être appréhendée sans tenir compte du fait que ce mouvement répond, avant tout, aux nécessités de l'accumulation du capital et du développement capitaliste.

\section{Concurrence et vitesse des communications}

Aux $15^{\text {ème }}$ et $16^{\text {ème }}$ siècles, la vitesse de rotation du capital n'était pas le seul élément d'articulation entre le développement capitaliste et celui de la vitesse des communications ; l'exercice concret de la concurrence contribuait également au développement et à l'accélération des communications. En effet, l'exercice de la concurrence capitaliste (i.e : pour l'accumulation du capital) se traduisait par la mise en concurrence des réseaux de communications, induisant ainsi une tendance générale au développement et à l'accélération des communications.

L'un des aspects majeurs des activités capitalistes consistait alors à gérer, le plus habilement possible, d'importantes variations de prix, aussi bien dans l'espace que dans le temps. Ces variations étant dans bien des cas imprévisibles, elles étaient porteuses d'un risque (au sens statistique du terme) important, du point de vue des hommes d'affaires. Or, concrètement, la "bonne" gestion de ce risque dépendait exclusivement de la capacité de chaque homme d'affaires à communiquer et traiter certaines informations, mieux et surtout plus vite que ses concurrents.

\section{$\underline{\text { Informations, prix et profits }}$}

À un instant donné, les prix d'un même produit pouvaient être très différents d'une ville à l'autre, d'une région à l'autre. En effet, les irrégularités de la production et surtout de la circulation, mais aussi la taille très réduite des marchés les plus lucratifs, rendaient l'offre extrêmement instable. Les pénuries locales ou régionales étaient fréquentes, d'abord pour les produits du commerce intercontinental, mais aussi pour les produits artisanaux ou industriels. Ainsi, au mois d'avril 1681, le cours des cuirs de Russie était presque deux fois et demi plus élevé à Livournes qu'à Amsterdam. Malgré le coût du transport, il y avait là une opportunité intéressante, mais à condition de la saisir avant tout le monde, c'est à dire avant que l'écart de prix ne se réduise. Pour cela, il fallait être informé de cet écart de prix, et acheminer la marchandise plus rapidement que les concurrents, c'est à dire, disposer du réseau de communications le plus performant et le plus rapide.

Mais surtout, les prix variaient dans le temps. Au delà des tendances longues, ils étaient soumis à des variations incessantes, et fréquemment en fonction d'évènements soudains et imprévisibles. En ce qui concerne les produits du commerce à long rayon d'action, les prix étaient entièrement dépendant du rythme d'arrivée des convois, dont on a vu à quel point il pouvait être irrégulier et imprévisible. Dès lors, du fait de la concurrence et de

\footnotetext{
${ }^{23}$ V. Vasquez de Prada. Op cit. Tome 1.
} 
la spéculation, ces prix étaient extrêmement volatiles, soumis à la moindre nouvelle ou rumeur.

Dans certaines grandes villes d'Occident, le phénomène touchait même les produits de base. Ainsi, à Venise, on apprit, le 3 janvier 1503, que les navires envoyés en Sicile par le marchand Stephan Contarini, afin de charger des blés, avaient trouvé les concessions d'exportation suspendues. Aussitôt, sur le marché vénitien, les cours du froment passèrent de 5 lires et 10 sous le staïo, à 6 lires et 4 sous. Puis, durant toute l'année 1503, les cours augmentèrent lentement, pour atteindre 6 lires et 12 sous, au mois de novembre. Mais, le 5 décembre, on apprit qu'un navire revenant de Chypre avec une cargaison de 5000 staïos de blé avait coulé. De nouveau on assista à une flambée des cours, qui montèrent jusqu'à 8 lires. $^{24}$

Sur les marchés plus spéculatifs où se concentraient les activités des hommes d'affaires, l'ampleur des variations de prix était encore plus grande. Ainsi, le 14 mars 1501, deux nobles vénitiens, faits prisonniers puis libérés par les Turcs, annoncèrent que Kamali, un corsaire turc, avait pris la mer avec une quarantaine de navires. Aussitôt, le taux d'assurance sur les galères effectuant le trajet Venise-Beyrouth passa de 1,5\% à 10\% de la valeur assurée. De la même façon, une lettre arrivée à Venise le 29 juin 1518, annonça que le navire Contarini Minotti avait été aperçu entouré de bateaux turcs, au large des Pouilles. Aussitôt la rumeur d'une nouvelle intensification de la piraterie se répandit, et les taux d'assurance grimpèrent. Mais finalement, deux jours plus tard, on apprit que le navire en question était arrivé à Bari sain et sauf. Au cours de la journée, les taux d'assurance retombèrent de $40 \%$ à $25 \% .^{25}$

On conçoit que dans un tel contexte, le fait de disposer d'une information susceptible de faire varier les prix, avant que le marché ne réagisse (i.e : avant que les concurrents n'en prennent connaissance), ait constitué une promesse de gains substantiels.

\section{$\underline{\text { Réseaux et vitesse des communications }}$}

C'est pourquoi les hommes d'affaires de la Renaissance s'attachèrent, avec une attention toute particulière, à mettre sur pied des réseaux de communication aussi sûrs et efficaces que possible, et se livrèrent, en la matière, une concurrence sans merci.

Dès les $15^{\text {ème }}$ et $16^{\text {ème }}$ siècles, les maisons commerciales dont le volume d'affaires rendait la chose possible, se dotèrent de véritables réseaux internationaux de collecte et de transmission des informations. Chaque grande compagnie disposait de représentations permanentes ou de succursales dans les plus grandes places commerciales européennes. Là, des informateurs et des courtiers avaient pour mission de collecter et de transmettre toutes les informations utiles à la maison mère, ainsi que de mettre en œuvre les décisions qui leur étaient communiquées.

Ainsi, au début du $16^{\text {ème }}$ siècle, la maison Welser d'Augsbourg entretenait des représentations à Nuremberg, Milan, Venise, Rome, Berne, Zurich, Fribourg, Lyon, Anvers, Lisbonne et Saragosse. ${ }^{26}$ De même, la maison Haug était présente à Anvers, Venise, Cologne, Nuremberg, Ulm et Schwarz dans le Tyrol. ${ }^{27}$ Plus généralement, parmi les autres maisons européennes de première importance ayant mis sur pied des réseaux internationaux de collecte des informations et de saisie des opportunités, on peut citer : Fugger (qui était représenté

\footnotetext{
${ }^{24}$ P. Sardella, 1948. Op cit.

${ }^{25}$ Ibid.

${ }^{26}$ P. Jeannin. Les marchands du $16{ }^{\text {ème }}$ siècle. Paris, Seuil, 1957.

${ }^{27}$ R. Ehrenberg. Le siècle des Fugger. Paris. S.E.V.P.E.N. 1955.
} 
jusqu'à Varsovie), Médicis, Ruiz, Mendez-Naci, Imhôf, Schetz, Veckinchusen, Güalterotti, Frescobaldi...

Entre les sièges des différentes maisons et leurs représentations, existaient des relations épistolaires permanentes, et d'un volume considérable. Au regard des moyens techniques de l'époque, la quantité d'informations circulant par les réseaux des hommes d'affaires, apparaît impressionnante. Ainsi, en seulement dix ans, entre 1395 et 1405, le siège d'un homme d'affaires florentin de second rang, Prato Francesco di Marco Datini, expédia ou reçut de ses succursales, représentations ou informateurs, plus de 80000 lettres. $^{28}$

Pour acheminer un tel volume de courrier dans des conditions acceptables, les grandes maisons commerciales furent, comme on l'a déjà mentionné, amenées à mettre sur pied, individuellement ou en se regroupant, leurs propres services postaux. Or, l'organisation et le fonctionnement de tels services coûtaient extrêmement cher ; bon nombre d'hommes d'affaires n'étant pas en mesure d'amortir de tels investissements, ils durent recourir, moyennant finances, aux services de sociétés spécialisées. L'examen rapide des tarifs d'une de ces sociétés, ${ }^{29}$ permet de se faire une idée concrète du prix que les hommes d'affaires accordaient à la vitesse de transmission de certaines informations.

Sur le trajet Venise-Beyrouth, par petit bateau express, les tarifs étaient en 1501, de 750 ducats vénitiens pour un voyage en 22 jours, 800 ducats pour 20 jours, et 850 ducats pour 18 jours. Sur le trajet Venise-Rome par courrier express, les tarifs étaient, en 1538, de 40 à 44 ducats pour 40 heures, de 35 à 40 ducats pour 44 heures, de 30 à 34 ducats pour 48 heures... Quand on sait que dans la Venise du début du $16^{\text {ème }}$ siècle, un ouvrier terrassier gagnait 13 ducats par an, un huissier d'administration 48, et le directeur de la perception des douanes 200, on appréhende mieux l'enjeu que pouvait constituer la vitesse des communications pour les hommes d'affaires de la Renaissance...

Le terme "vitesse" doit cependant être précisé. Ce que recherchent les hommes d'affaires, ce n'est pas la vitesse des communications en tant que telle. S'il sont prêts à payer si cher, ce n'est pas tant pour communiquer vite, que pour communiquer plus vite que les concurrents, car, comme on l'a vu, c'est de cette différence éventuelle que dépend la réalisation de profits exceptionnels. Dès la fin du $14^{\text {ème }}$ siècle, un homme d'affaires italien, Paolo di messer Pace da Certaldo, illustre, on ne peut plus clairement, le caractère "concurrentiel" de la vitesse des communications : ${ }^{30}$

"Si tu fais du commerce, et si aux lettres qui te sont destinées sont jointes, lorsqu'elles te parviennent, des lettres destinées à autrui, aie toujours à l'esprit de lire d'abord tes lettres avant de remettre celles d'autrui. Et si tes lettres contiennent des conseils d'achat ou de vente qui doivent tourner à ton bénéfice, convoque aussitôt le courtier, et fais ce que te conseillent tes lettres, et ensuite seulement remets les lettres qui sont venues avec les tiennes. Mais ne les remets jamais avant d'avoir d'abord fait tes affaires, car ces lettres pourraient contenir des indications qui iraient à la traverse de tes affaires, et le service que tu aurais rendu à un ami, à un voisin ou à un étranger de lui porter ses lettres tournerait à ton grand détriment : or, tu ne dois pas servir autrui pour te desservir dans tes propres affaires. »

\footnotetext{
${ }^{28}$ J. Favier. De l'or et des épices. Naissance de l'homme d'affaires au Moyen Âge. Paris, Fayard, 1987.

${ }^{29}$ P. Sardella, 1948. Op cit.

${ }^{30}$ Cité par : Y. Renouard. Les marchands italiens du Moyen Âge. Paris, Armand Colin , 1949, p 181.
} 


\section{Concurrence et accélération des communications}

À l'époque du capitalisme commercial, l'efficacité et la vitesse des communications apparaissent donc comme l'un des objets essentiels de l'exercice de la concurrence capitaliste.

"Faire connaître à son siège s'il [le marchand] est en poste éloigné, aux succursales ou filiales s'il est au siège, en un point de convergence de l'information, les usages qui s'établissent et les goûts qui changent, ce que veut la clientèle et ce qu'offre le marché, comme notifier le prix du poivre ou du fil d'or, mander d'urgence le taux des changes, mesurer avant d'autres les flux monétaires et annoncer le premier l'arrivée d'un navire ou le naufrage d'un autre, c'est le moyen de peser sur le marché monétaire et sur celui des denrées, de jouer des opportunités et de devancer la concurrence. La prime est ici à celui qui dispose du meilleur réseau, des relations les mieux placées, des communications les plus rapides. Elle est à celui qui sait avant d'autres, ce que sera demain la guerre ou la paix, la disette ou l'abondance. ${ }^{31}$

Or, de cette concurrence sans merci en matière de vitesse des communications découle, par nature, une tendance générale (macro-économique) à l'accroissement de cette vitesse. Dans cette perspective, l'accélération des communications apparaît comme un mouvement au moins partiellement induit par l'exercice de la concurrence, c'est à dire, [au moins partiellement] dépendant de la dynamique d'accumulation du capital.

\section{Pour une perspective de longue durée}

On s'est efforcé, à travers les brèves considérations qui précèdent, de mettre en évidence le fait que l'évolution de la vitesse des communications à la Renaissance ne peut être analysée et comprise sans recourir, à un moment ou à un autre, à une approche en termes de longue durée, c'est à dire, à une approche qui privilégie les tendances longues de l'évolution de la vitesse des communications, ainsi que leurs interférences avec les mouvements de longue durée moteurs de l'évolution sociale.

Plus concrètement, on a montré, qu'à l'époque étudiée, et dans une perspective de longue durée, l'accélération des communications apparaît comme un mouvement à la fois, induit par le développement capitaliste, et contribuant à ce développement.

D'une part, on a vu que dans les conditions historiques considérées, la tendance à l'accélération de la rotation du capital, inhérente au développement capitaliste, se traduit matériellement par une tendance à l'accélération des communications ; il en va de même pour la concurrence, dont l'exercice concret (micro-économique) se traduit par une tendance générale (macro-économique) à l'accélération des communications, ainsi, d'ailleurs, qu'au développement des réseaux. D'autre part, on a constaté que dans le cadre du capitalisme commercial, toute accélération des communications rend possible un accroissement de la vitesse de rotation du capital, et contribue ainsi au développement de l'accumulation du capital.

Il apparaît donc finalement que, dans le cadre historique retenu, l'évolution (de longue durée) de la vitesse des communications apparaît très largement conditionnée par les nécessités du développement capitaliste.

\footnotetext{
${ }^{31}$ J. Favier, 1987. Op cit. p 83.
} 
Bien sûr, ces quelques constatations ne devraient pas être directement utilisées pour éclairer, dans une perspective de longue durée, la problématique des mouvements actuels de l'articulation procès de communication-procès d'accumulation du capital. On ne saurait, sans précautions, passer d'un détail à l'ensemble du "tableau". D'un autre côté, il ne saurait être question de justifier en quelques lignes une telle généralisation des résultats. On se contentera donc de caractériser cette généralisation, en signalant qu'elle repose sur des résultats et des principes méthodologiques de l'analyse des mouvements économiques de longue durée qu'il est impossible de détailler ici. ${ }^{32}$

On considèrera tout d'abord, que l'observation selon laquelle l'accélération des communications est conditionnée par les nécessités du développement capitaliste est valable non seulement pour les débuts du capitalisme commercial, mais aussi, beaucoup plus largement, pour l'ensemble de la période caractérisée par la prépondérance du procès d'accumulation du capital dans le mouvement social, c'est à dire, pour l'ensemble du capitalisme. Cela n'exclut pas, bien sûr, que des hommes d'affaires de la Renaissance au mode d'accumulation fordiste, les modalités de la relation vitesse des communications-accumulation du capital se soient profondément transformées, au gré des transformations du mode d'accumulation ; mais on supposera que la nature de cette relation, (i.e : l'évolution de la vitesse des communications conditionnée par l'accumulation du capital) est, elle, restée inchangée. Non pour justifier mais pour illustrer très grossièrement cette "hypothèse", on peut indiquer qu'elle revient, par exemple, à considérer que l'accélération de la vitesse des communications au $19^{\text {ème }}$, puis au $20^{\text {ème }}$ siècle, n'a de sens que dans le cadre de la Révolution Industrielle, puis de l'essor du fordisme. On concèdera, qu'au delà des problèmes méthodologiques posés par cette généralisation des résultats, une telle "hypothèse" n'est pas, $a$ priori, totalement irréaliste.

Le second aspect de la généralisation des résultats consiste à étendre les observations réalisées sur la vitesse des communications à l'ensemble du procès de communication. Bien entendu, une telle extension apparaît, a priori, plus que douteuse sur le plan méthodologique ; on remarquera cependant, qu'il ne s'agit pas d'en déduire des résultats ou des conclusions, mais d'en tirer quelques interrogations destinées à fournir l'ébauche d'une problématique. En tout état de cause, pour tenter d'indiquer quelques axes très généraux de ce que pourrait être une approche en termes de longue durée des mouvements actuels du procès de communication, on considérera que, tant que domine la dynamique du capitalisme, les mouvements du procès de communication ne sont pas autonomes, et gravitent autour du mouvement déterminant de l'évolution sociale qu'est le développement de l'accumulation du capital.

On peut alors, tenter de préciser quelque peu la portée des diverses interprétations possibles du développement contemporain du procès de communication. En laissant de côté l'interprétation selon laquelle ce développement ne serait qu'anecdotique, du point de vue de la longue durée, on se retrouve finalement devant l'alternative suivante : les mouvements actuels du procès de communication s'inscrivent-ils dans une crise de la logique même d'accumulation du capital, c'est à dire, dans une crise du capitalisme, ou participent-t-ils d'une crise du mode d'accumulation, c'est à dire, des modalités de mise en œuvre de la logique d'accumulation du capital ?

Évoquer l'émergence d'une "société de communication", c'est, au moins implicitement, considérer que les mouvements contemporains du procès de communication sont non seulement autonomes, mais en outre, porteurs d'une logique et d'une dynamique propres, potentiellement contradictoires avec celles de l'accumulation du capital ; à travers la crise

\footnotetext{
${ }^{32}$ S. Walery, 1987. Op cit.
} 
contemporaine, les mouvements du procès de communication cesseraient progressivement d'être conditionnés par la dynamique de l'accumulation du capital, pour entrer en "concurrence" avec elle, et devenir peu à peu un élément moteur de l'évolution sociale. C'est donc la nature même de l'articulation communication-accumulation du capital propre au capitalisme qui se trouverait mise en cause, et, à travers elle, la dynamique du capitalisme elle-même.

L'autre interprétation consiste à considérer que, tout en continuant de graviter autour de la dynamique d'accumulation du capital, les mouvements contemporains du procès de communication s'inscrivent dans le cadre de la crise (i.e : de la redéfinition) du mode d'accumulation. Dans une telle perspective, on peut, soit supposer que comme depuis cinq siècles, les transformations du procès de communication sont un élément, parmi d'autres, de l'évolution des modalités d'accumulation du capital, soit attribuer aux activités de communication un rôle spécifique dans cette crise, en considérant qu'elles sont appelées à devenir un élément central du nouveau mode d'accumulation en gestation.

Pour esquisser une illustration de cette alternative, on peut l'appliquer, très brièvement, à l'élément du procès de communication qui a fait l'objet des quelques pages qui précèdent.

Dans le cas d'une crise du capitalisme liée à l'émergence d'une "société de communication", on pourrait imaginer, que l'accélération considérable des communications à laquelle on assiste aujourd'hui, et qui, dans certains cas, va jusqu'à l'instantanéité, n'est plus désormais en phase avec l'accroissement de la vitesse de rotation du capital et l'exercice de la concurrence. Par exemple, le degré atteint en matière de vitesse des communications serait déjà tel, que les progrès futurs ne pouvant être que marginaux, le potentiel d'accroissement de la vitesse de rotation du capital s'en trouverait désormais très réduit ; certains seraient également enclins à penser, que le développement des communications en temps réel prive peu à peu d'objet, l'exercice de la concurrence en matière de vitesse des communications, tendant ainsi à dénaturer l'un des moteurs essentiels de la dynamique du capitalisme.

Dans l'autre perspective, on considèrera au contraire, que les progrès de la vitesse des communications, même s'ils s'inscrivent et peut-être de "façon centrale", dans le cadre d'une redéfinition des modalités de l'accumulation du capital, ne mettent fondamentalement en cause ni le potentiel d'accroissement de la vitesse de rotation du capital, ni l'exercice de la concurrence. Dans ce cadre, on remarquera, bien sûr, que la vitesse de rotation du capital ne dépend pas seulement de la vitesse des communications ; on soulignera que le développement des techniques en temps réel n'a pas encore aboli toute possibilité de concurrence en matière de vitesse de collecte de transmission et de traitement des informations, ainsi que d'élaboration et surtout de mise en pratique ou de concrétisation des décisions.

Bien sûr, une telle alternative intéresse aussi bien les économistes que les spécialistes de la communication. Y répondre rigoureusement exige qu'à un moment ou à un autre, les uns et les autres trouvent un terrain commun pour confronter les résultats de leurs réflexions.

Du point de vue des spécialistes de la communication il conviendra de s'intéresser en priorité aux activités du procès d'accumulation du capital qui apparaissent les plus directement concernées par l'accélération contemporaine des communications. On pense, bien sûr, aux activités financières, "secteur" où désormais, du fait de la vitesse des communications, la vitesse potentielle de rotation du capital semble tendre vers l'instantanéité, et où la marge de concurrence paraît de plus en plus infime.

Du point de vue des économistes, qui, par nature, sont bien peu enclins à envisager l'éventualité d'une crise du capitalisme, on remarque cependant une attention toute particulière 
pour le développement considérable de la "sphère" financière auquel on assiste depuis quelques années. Pour ceux qui perçoivent, dans la crise actuelle, une transformation du mode d'accumulation du capital, les activités financières apparaissent souvent comme un champ d'analyse privilégié, pour tenter de mettre à jour les caractéristiques du mode d'accumulation en gestation. $^{33}$

Pour peu qu'elle se réfère à la problématique de longue durée, l'étude des activités financières apparaît ainsi, malgré les différences de perspective, comme un des points de rencontre possibles entre économistes et spécialistes de la communication, pour tenter de mieux comprendre le devenir de l'articulation communication-accumulation du capital.

Pour finir, on rappellera que dans une perspective de longue durée, aucune des éventualités rapidement mentionnées ci-dessus n'est, a priori, contestable. Cela s'applique en particulier à l'hypothèse de l'émergence d'une "société de communication". Cependant, jusqu'à présent, les différents discours sur le procès de communication ignorant totalement la perspective de longue durée, aucune justification cohérente n'a été proposée, en particulier par ceux qui proclament la naissance d'une "société de communication", d'où le malaise que provoque inévitablement ce terme, et l'ambigüité inhérente aux propos qui s'y réfèrent.

Pour paraphraser Fernand Braudel, ${ }^{34}$ serait-ce trop demander à tous ceux qui, dans le cadre de leurs recherches, sont amenés à s'intéresser au procès de communication, que de temps à autre, ils confrontent leurs résultats à la perspective de longue durée, afin de contribuer à déterminer de façon rigoureuse, le devenir des relations entre communication et accumulation du capital. En retour, cette confrontation les aiderait à mieux prendre conscience de la presque immobilité des temps longs du mouvement social, ainsi qu'à mieux relativiser le scintillement de l'évènementiel et les mirages de la technique. Dans cette optique, et sans volonté polémique, on ne peut résister à la tentation de rappeler, que dès les $14^{\text {ème }}$ et $15^{\text {ème }}$ siècles : ${ }^{35}$

"[L'homme d'affaires italien] est un personnage qui dirige de son bureau, avec ses livres de comptes et les informations que lui adressent tous ses correspondants et que lui apportent les courriers, des affaires réparties sur un échiquier international et parfois mondial, sans manipuler lui-même ni les marchandises, ni les espèces qui en sont l'objet.»

Pour certains, une telle observation pourrait suffire à considérer, qu'à ce jour, il n'est pas totalement déraisonnable de conserver un embryon de scepticisme quant aux bouleversements du mouvement social dont seraient porteuses les techniques contemporaines d'information et de communication.

\footnotetext{
33 À cet égard, voir en particulier l'évolution générale de l'orientation des travaux de Michel Aglietta depuis une quinzaine d'années.

${ }^{34}$ "La longue durée nous paraît la ligne la plus utile pour une observation communes aux sciences sociales. Est-ce trop demander à nos voisins [économistes, sociologues...] que de souhaiter qu'à un moment de leurs raisonnements, ils ramènent à cet axe leurs constatations ou leurs recherches ?».

F. Braudel, 1958. Op cit.

${ }^{35}$ Y. Renouard. Op cit. p 175.
} 